\section{The experiences of clinic treatment of a severe case of novel influenza virus $A$ (H1N1) infection}

\author{
Jun Zhang, ${ }^{1}$ Guangdan Zhao, ${ }^{1}$ \\ Xiaoling Yu, ${ }^{1}$ Jiaying Sun, ${ }^{1}$ \\ Xiao Ming Pan, ${ }^{1}$ Yong Gang $\mathrm{Li}^{2}$ \\ 'Department of Respiratory Diseases, \\ The Forth Hospital affiliated to China \\ Medical University; ${ }^{2}$ Department of \\ Virology, Research Institute for Microbial \\ Diseases, Osaka University, Osaka, Japan
}

\section{Abstract}

We present here a case of cough, expectoration and fever for six days. After investigation by the doctor and epidemiologist, it was confirmed that this patient had a history of contact with a novel influenza virus. All the results of the real time PCR on novel Swine Influenza H1 were positive. Blood-gas assay $\mathrm{PO}_{2} 50.6 \mathrm{mmHg}$, hypoxemia and computed tomography (CT) of lungs indicated patchy dense shadow scattered in both lungs in which the inflatable bronchial shadow was observed. A visible change in leakage around the shadow was seen. This was a serious case of infection from a novel influenza virus and the patient received systemic treatment: oseltamivir $75 \mathrm{mg}$ bid po, methylprednisolone $40 \mathrm{mg}$ qd iv, biapenem $0.6 \mathrm{q} 12 \mathrm{~h}$ iv, and moxifloxacin 0.4qd po. On discharge from hospital, Swine influenza H1 was negative. Lungs showed apparent absorption of the inflammation. Respiratory failure had been corrected. Patients infected with a novel influenza virus should be given low-dose hormone and an antiviral drug as soon as possible after the infection is confirmed.

\section{Case Report}

A fifty-eight year old male presented with cough, expectoration and fever for six days. After investigation by the doctor and epidemiologist, it was confirmed that this patient had a history of contact with a novel influenza. Before coming to our hospital, this patient had been infused at the Community Hospital for three days. Since there had been no change in his condition, the patient was admitted to our emergency room. Body temperature was $39.5^{\circ}$, respiratory frequency was 20 times/min. There was lassitude, superficial lymph nodes were swollen, while nasal ventilation was good. There was no abnormal secretion, throat congestion, or bilateral antiadoncus. There was no exudation and lips were cyanosis. Immediate percussion of lungs showed vesicular resonance, and small and middle bubbles were heard in the back at the bottom of the lungs. Pharynx swabs were taken and sent to the Control Disease Center (CDC) when the patient was hospitalized. Computed tomography (CT) of lungs indicated patchy dense shadow scattered in both lungs, in which the inflatable bronchial shadow was observed. The boundary of shadow was not clear. A visible change in leakage around the shadow was seen (Figure 1). On the third day after hospitalization, another CT indicated that high density shadow had apparently increased in both lungs and diffused to the individual lung lobe (Figure 2). A routine blood test was urgently checked; white blood cells were $3.59 \times 10^{9} / \mathrm{L}$, neutrophils 0.641 , lymphocytes 0.2674 , monocytes 0.0864 , hemoglobin $145 \mathrm{~g} / \mathrm{L}$, platelets $135 \times 10^{9} / \mathrm{L}$, blood-gas assay $\mathrm{PO}_{2} 50.6 \mathrm{mmHg}$, oxygenation index $230 \mathrm{mmHg}$, random blood glucose assay, $11.39 \mathrm{mmol} / \mathrm{L}$. Routine screening of feces and urine, and biochemical blood tests, such as liver function, renal function, myocardial enzymes, blood lipid, and electrolytes showed no obvious abnormalities. Based on the course of the illness and the clinical symptoms, this patient was newly diagnosed as a suspicious influenza virus case. All the results of the real time PCR on novel Swine Influenza $\mathrm{H} 1$ were positive. The experiment was carried out by the Liaoning Province Center for Disease Control and Prevention (http://www.lncdc.com/\#; data not shown). This patient was confirmed a severe novel influenza A case. After being admitted into hospital, he was newly diagnosed with suspicious novel influenza infection and was admitted to a single isolated room and given treatment: bed rest, increased water consumption, oxygen mask therapy, oseltamivir (Kewei Yichang Changjian Pharmaceutical Co., Ltd.) $75 \mathrm{mg}$ bid po, methylprednisolone (America Pharmaceu-tical Co., Ltd.) 40mg qd iv, biapenem (Nanjing Xiansheng Dongyuan Pharmaceutical Co., Ltd) 0.6 q12h iv, moxifloxacin 0.4qd po, glarginen insulin (SannofiAventis Deutschland $\mathrm{GmbH}$ ) to control blood glucose. After two weeks of treatment, a further lung CT was taken which showed that the lung inflammation had apparently been absorbed (Figure 3). Blood gas assay showed $\mathrm{PO}_{2} 82.6 \mathrm{mmHg}$. The patient was considered cured and was discharged.

\section{Discussion}

Novel swine-origin influenza A (H1N1) virus, more commonly known as swine flu,
Correspondence: Jun Zhang, Department of Respiratory Diseases, The Fourth Hospital affiliated to China Medical University, 102 Nan Qi Road, He Ping District, Shen Yang City, Liao Ning Province, China 110005.

Tel. +86.24.6203.6327.

E-mail: cnzhangjun655@hotmail.com

Key words: novel swine-origin influenza A, H1N1, hormone, antiviral, treatment.

Received for publication: 12 October 2011. Revision received: 31 0ctober 2011. Accepted for publication: 2 November 2011.

This work is licensed under a Creative Commons Attribution NonCommercial 3.0 License (CC BYNC 3.0).

CC Copyright J. Zhang et al., 2011

Licensee PAGEPress, Italy

Microbiology Research 2011; 2:e23

doi:10.4081/mr.2011.e23

was first reported in Mexico in April 2009. Since then it has rapidly spread to many countries around the world. Novel influenza A (H1N1) is a new swine-origin virus and has become the current dominant strain. In June 2009, the WHO declared the emergence of a global pandemic, raising the alert level to phase 6 (pandemic phase). With the development of the epidemic situation, the number of severe cases and deaths increased.

Clinical manifestations are varied and include fever, cough, sore throat, body aches, headache, chills and fatigue. In addition, nausea, vomiting, and/or diarrhea have also been reported.

The infection occasionally leads to severe primary viral pneumonia leading to acute respiratory distress syndrome, multiple organ dysfunction and death. Most medical doctors have experience of the diagnosis and treatment of novel influenza A (H1N1) infection. Developments in the prevention and control of influenza virus have been very promising. The aim of this paper is to report the experience of a severe influenza A (H1N1) case who was successfully cured.

\section{Change in epidemiological characteristics}

By June $11^{\text {th }}, 2009$, nearly 30,000 case of 2009 H1N1 virus had been confirmed across 74 countries, compelling the WHO to signal the phase 6 alert level. ${ }^{1}$ At the beginning of the outbreak of influenza A (H1N1), most of the patients had clear contact history or had even traveled to the epidemic area. However, in China, aggregation epidemic events had been happening frequently since September 2009 , especially after the long holiday of 
National Days. The epidemic situation developed rapidly. The number of undiagnosed cases and asymptomatic cases increased. The contact history of many cases was not clear. Doctors in outpatient clinics and emergency units had to be highly vigilant to these characteristic changes and not depend only on the contact history. Cases have changed from outbreaks in schools to mainly sporadic outbreaks in the community. This has not been conducive to epidemic prevention and control.

\section{Early diagnosis can improve the prognosis of this infection}

Careful attention and an understanding of this disease could lead to an early diagnosis and therapy. This is the key for a good prognosis. Clinicians had been advised that compli- cations from the $2009 \mathrm{H} 1 \mathrm{~N} 1$ virus were similar to those of the seasonal influenza virus. ${ }^{2}$ As we know, pathological features of respiratory virus infection mainly concern lung damage accompanied by multi-organ disease. A review of 44 confirmed $2009 \mathrm{H} 1 \mathrm{~N} 1$ cases in a New York City high school revealed that cough (98\%), fever (96\%), headache (82\%), sore throat $(82 \%)$, rhinorrhea $(82 \%)$, chills $(80 \%)$, and muscle aches $(80 \%)$ were reported. ${ }^{3}$ Lung damage was mainly shown as a diffusion in lung and an alveolar damage which went through the exudation period, proliferative phase and the fibrotic stage. The key point which can affect prognosis is in the phase of the exudation period. Usually, exudation takes place in the first week of the disease. Edema, hemorrhage and bleed have been seen in the lung tissue. Liquid of protein edema and inflammation cell invasion were seen in the pulmonary interstitial and lung alveolar which gradually developed to plasma protein coagulation. The occurrence of pulmonary edema and the formation of the transparent membrane are also accompanied by pulmonary interstitial fibrosis (PIF). All these changes can promote respiratory failure. CDC examined postmortem lung specimens from patients with fatal cases of 2009 pandemic influenza A (H1N1) to evaluate the role of bacterial coinfection in fatal outcomes of H1N1 infection. ${ }^{4}$ Patients who had bacteria coinfection present with fever, dyspnea, cough, and abnormal chest X-rays., Treatment in the first week is a critical factor in deciding disease prognosis. CT imagery will be useful to check the dynamic monitoring of changes in the lungs.
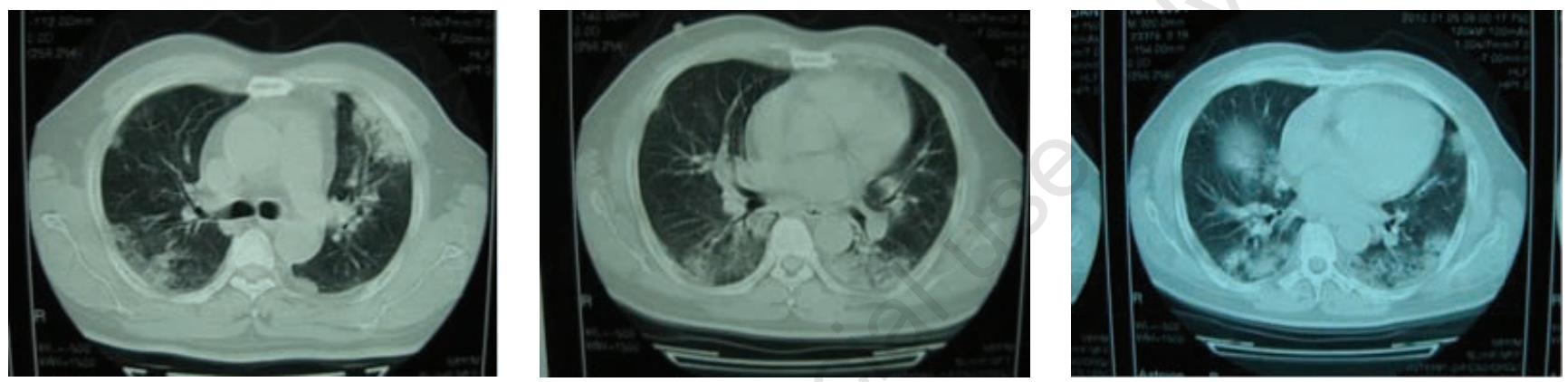

Figure 1. Lung computed tomography. Lung computed tomography indicated a patchy dense shadow scattered in both lungs, in which the inflatable bronchial shadow was observed. The boundary of shadow was not clear. Visible change in leakage around shadow was seen.
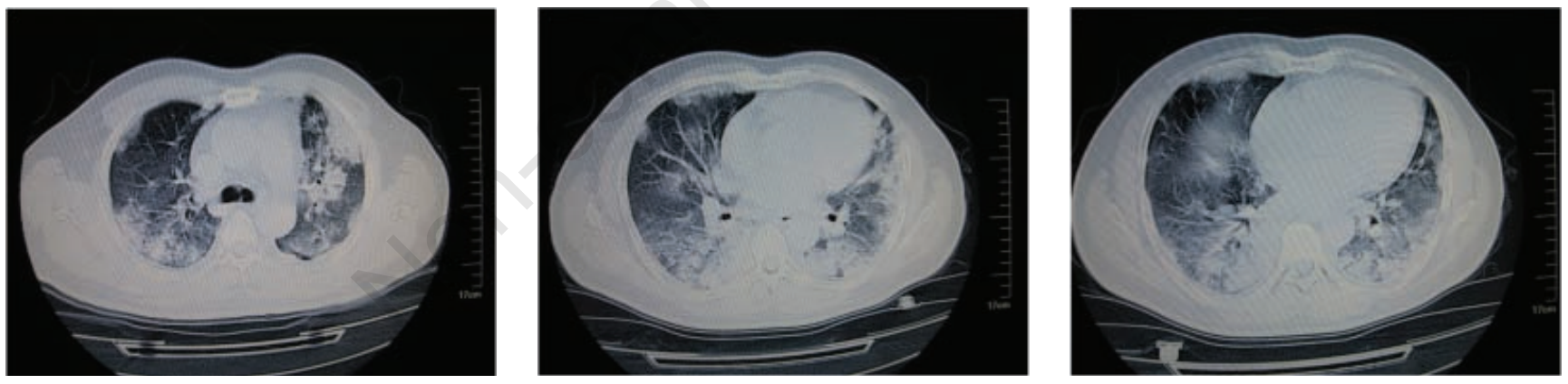

Figure 2. Lung computed tomography. A high density shadow apparently increased in both lungs and diffused to individual leaf of both lungs.
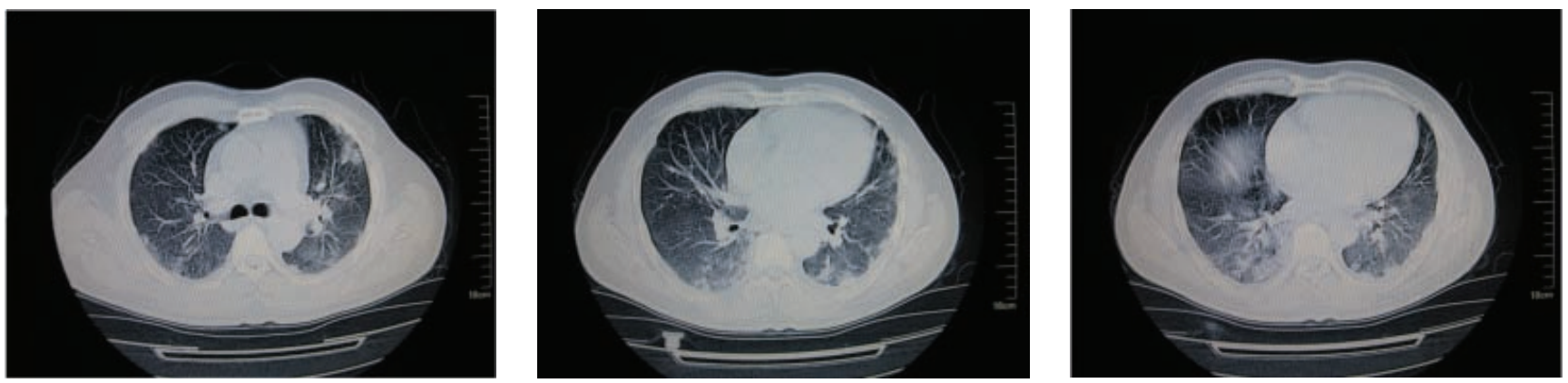

Figure 3. Lung computed tomography. Apparent absorption of lung inflammation. 


\section{Early application of low-dose hormone}

The mechanisms of action of glucocorticoid are: anti-inflammatory, immunosuppressive, anti-shock, hematopoietic stimulation and excitability. The anti-inflammatory effect can reduce inflammation exudation, edema and invasion of the inflamed cells. Since the first severe acute respiratory syndrome (SARS), glucocorticoid application has been helpful. Almost all respiratory disease specialists have affirmed the critical role of hormones in the rescue program for patients with severe disease. Professor Nanshan Zhong indicated that application of glucocorticoid can reduce the exudation damage and subsequent pulmonary fibrosis. It can improve the patient's general clinical condition. ${ }^{2}$ Glucocorticoid is essential for the treatment of the severe influenza A (H1N1) patient. However, careful attention must be given to ensure that glucocorticoid is not a double-edged sword. It can bring about severe side-effects (femoral head necrosis and pulmonary fibrosis). Hormone therapy should be strictly controlled according to the patient's condition. Large doses of hormone should not be administered. For the patient with severe disease, duration of treatment of high-dose hormone therapy should not be too long. We recommend that methylprednisolone $40-80$ $\mathrm{mg} /$ day for 7-10 days are suitable.

\section{Rational use of antiviral therapy}

Given the reference from the sequences of neuraminidase (NA) of this virus, this novel influenza virus A (H1N1) remains susceptible to NA inhibitors ${ }^{7,8}$ oseltamivir and zanamivi. Because oseltamivir and zanamivir have proven efficacy in the treatment of human influenza when started in the early phase of the illness, it is better to give the patient an anti-virus drug as soon as possible. Oseltamivir should be given during the first 48 $h$ of fever onset. More specifically, the best time is during the first $36 \mathrm{~h}$ of onset. Treatment must continue for five days. Studies have suggested that hospitalized patients benefit from treatment initiated even later. ${ }^{9,10}$ At a late stage of the illness, patients often get secondary infection.

Most infections are due to complications. We recommend that antibiotics should be used as soon as the patient has been diagnosed with secondary bacterial infection. A greater understanding of H1N1 will mean we can know more about the clinic problems of this infection. Here, we have shared our personal experiences of the successful management of a severe case of H1N1 infection. We hope this information can provide a point of reference and help other doctors in their treatment of severe influenza virus infection. Influenza infection is not only preventable and controllable, but can also be cured.

In summary, we believe that early diagnosis is important and the patient infected with novel influenza virus should be treated with suitable hormone and antiviral drugs as soon as possible.

\section{References}

1. Word Health Organization. Current WHO phase of pandemic alert. Available from: http://www.who.int/csr/disease/livianinfluenza/phase/en//index.html. Accessed November 10, 2009.

2. Centers for Disease Control and Prevention (CDC). Interim guidance for clinicians an identifying and caring for patients with swine-origin influenza A (H1N1) Virus infection. Available from: www.cdc.gov/hlnl flu/identifying patients. htm. Accessed November 11, 2009.

3. Centers for Disease Control and Prevention (CDC). Swine-origin influenza A (H1N1) Virus infections in a school-New York City, April 2009. MMWR Morb Mortal Wkly Rep 2009;58:470-2.

4. Centers for Disease Control and Prevention (CDC). Bacterial coinfections in lung tissue specimens from fatal cases of 2009 pandemic influenza $\mathrm{A}(\mathrm{H} 1 \mathrm{~N} 1)$-united states, May-August 2009. MMWR Morb Mortal Wkly Rep 2009;58:1071-4.

5. Rorthberg MB, Haessler SD, Brown RB. Complications of viral influenza. Am J Med 2008;121:258-64.

6. Treanor JJ. Influenza Virus. In: GL Mandell, JE Bennett, R Dolin (eds.) Mandell, Douglas, and Bannett's Principles and Practice of Infectious Diseases. 6th ed. Philadelphia, PA, Elsevier Churchill Livingstone. 2005:2060-85.

7. Itoh Y, Shinya K, Kiso M, et al. In vitro and in vivo characterization of new swine-origin H1N1 influenza Virus. Nature 2009; 460:1021-5.

8. Leung TWC, Tai ALS, Cheng PKC, et al. Detection of an oseltamivir-resistant pandemic influenza A/H1N1 virus in Hong Kong: J Clin Virol 2009;46:298-9.

9. Hanshaoworakul W, Simmerman JM, Narueponjirakul U, et al. Severe human influenza infections in Thailand: Oseltamivir treatment and risk factors for fatal outcome. Plos ONE 2009:4:e6051.

10. McGeer A, Green KA, Plevneshi A, et al. Toronto Invasive Bacterial Diseases Network. Antiviral therapy and outcomes of influenza requiring hospitalization in Ontavio Canada. Clin Infect Dis 2007:45: 1568-75. 